\title{
The International Astronomy Olympiad
}

\author{
Michael G. Gavrilov \\ ISSP of Russian Academy of Sciences, \\ Institute street 2, 142432 Chernogolovka, Moscow region, Russia \\ email: gavrilov@issp.ac.ru
}

\begin{abstract}
The International Astronomy Olympiad (IAO) is an annual scientific-educating event for students of the junior high-school classes, aged 14-18 years. The Euro-Asian Astronomical Society founded the IAO in 1996. The Olympiad includes an intellectual competition between these students. The style of the problems is aimed at developping the imagination, creativity and independent thinking.
\end{abstract}

Keywords. Education, teaching, olympiad

\section{General}

- The International Astronomy Olympiad (IAO) is a scientific-educating event for students of the junior high school classes.

- Age of the contestants 14-18 years old.

- The main activity at the IAO: an intellectual competition between these students.

- Teams of different countries participate.

- Team from a country: 5 students and 2 team leaders, one of the team leaders is jury member.

\section{Aims}

There are very many points in the aims, in short, they are in branches of:

- Spreading astronomical knowledge among as many students as possible.

- Improving the education in astronomy and related sciences.

- Attracting the most talented young people to professional careers in the field of astronomy.

- Stimulating the imagination and creativity of children.

- Cultivating a spirit of correctness and friendship.

\section{Rounds}

The main events during the Olympiad's rounds. There are 3 rounds :

(a) Theoretical round - solving problems. The style of the problems is aimed at developing the imagination, creativity and independent thinking. See example of the problems below.

(b) Observational round - questions based on observing the sky and (maybe) working with telescopes.

(c) Practical round - working with the experimental (observational) data. 


\section{Syllabus}

- A considerable part of the knowledge which is necessary for participation in the Olympiad goes beyond the frames of the school curriculum in astronomy in most of the countries in the world.

- But as usual no additional knowledge in mathematics and physics is necessary.

- So preparation for the Olympiad needs extra-curricular activities in astronomy itself. These activities may be of various types, all of them promote astronomy education in the participating countries.

\section{Results}

According the rank the participating students are awarded by:

- Diploma of the I degree (gold medal).

- Diploma of the II degree (silver medal).

- Diploma of the III degree (bronze medal).

- Diploma of participation.

Additionally:

- Special Diploma (for the best result, best result in every round, original solution, best picture, etc.).

\section{Founding}

IAO was founded on June 7, 1996 by Euro-Asian Astronomical Society. The International Astronomy Olympiad became the sixth International Science Olympiad alongside with the Olympiads on Mathematics, Physics, Chemistry, Informatics and Biology.

\section{History}

The 1st IAO was held at the Special Astrophysical Observatory, Russia, in November 1996

\section{List of Olympiads}

(a) 1996 1st IAO, SAO RAS, Russia, 4 countries.

(b) 1997 2nd IAO, SAO RAS, Russia, 4 countries.

(c) 1998 3rd IAO, SAO RAS, Russia, 5 countries.

(d) 1999 4th IAO, Crimea, Ukraine, 7 countries.

(e) 2000 5th IAO, SAO RAS, Russia, 8 countries.

(f) 2001 6th IAO, Crimea, Ukraine, 7 countries.

(g) 2002 7th IAO, SAO RAS, Russia, 11 countries.

(h) 2003 8th IAO, Stockholm, Sweden, 13 countries.

(i) 2004 9th IAO, Crimea, Ukraine, 18 countries.

(j) 2005 10th IAO, Beijing, China, 15 countries.

(k) 2006 11th IAO, Bombay, India, 16 countries.

(l) 2007 12th IAO, Crimea, Ukraine, 23 countries.

(m) 2008 13th IAO, Trieste, Italy, 19 countries.

(n) 2009 14th IAO, Hangzhou, China, 17 countries

(o) 2010 15th IAO, Crimea, Ukraine, 


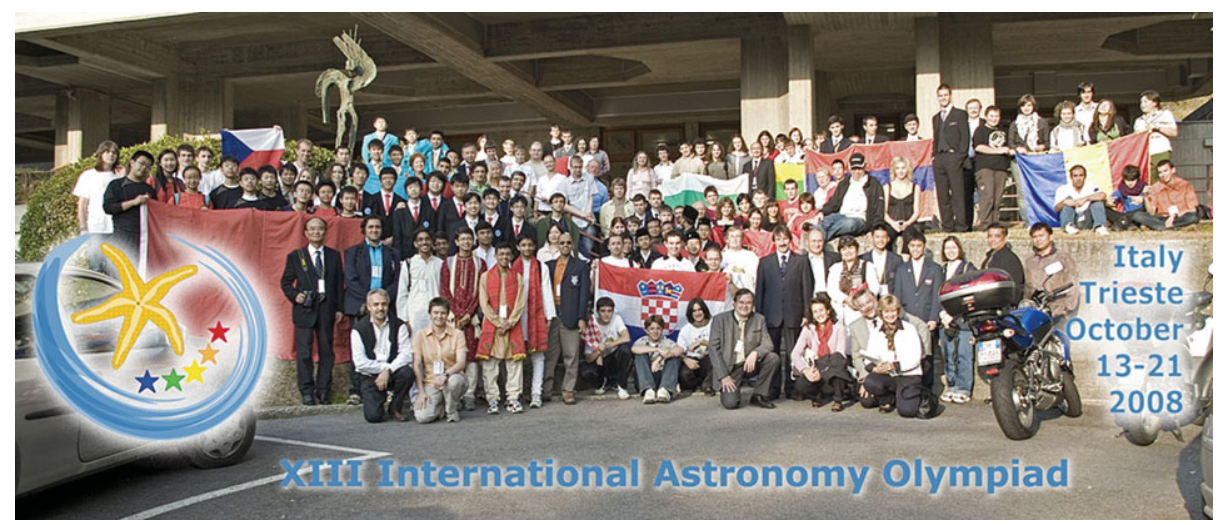

Figure 1. Participants of the XIIIth International Astronomy Olympiad.

\section{Participating countries}

In the past few years about 20 countries participated in the IAO. Traditionally participating countries are: Armenia, Brazil, Bulgaria, China, Crimea, Croatia, Czechia, Estonia, India, Indonesia, Iran, Italy, Kazakhstan, Korea, Lithuania, Moscow, Romania, Russia, Serbia, Sweden, Thailand, Ukraine.

Some countries participate irregularly. We hope that the number of the countries will be much larger in the near future.

\section{Olympiads" "daughters"}

- The Asian-Pacific Astronomy Olympiad has been held 4 times since since 2005 (in Irkutsk, Vladivostok, Xiamen and Bishkek). The 5th APAO (2009) was hold in Damyang (Korea), while the 6th APAO will take place in Karubara (Indonesia) in 2010.

- European Astronomy Olympiad has been held one time (2003, Stockholm). Organisers plan to restarted it in 2011 in format of JENAO (Joint European-National Astronomy Olympiad).

- New Independent States Astronomy Olympiad has been held 11 times in Special (North Caucasus, Russia), Crimean (Crimea, Ukraine) and Byurakan (Armenia) Astrophysical Observatories. Founded in 1990. Annually organised since 2003.

\section{What is important}

The IAO is an educational event. The problems of IAO teach the students not only to observe our Universe, not to learn only facts, but to think about the nature of the astronomical events.

The jury at IAO consists of professional astronomers who check in the students' solutions, at first the approach to the problem and not only the formal answer.

Students who arrive to IAO are the most talented students in astronomy of their countries. Communication between such students from young ages is very important for the future collaboration between nations.

At the Olympiad favourable conditions are created for active contacts between teachers and specialists from the different countries. We have intensive exchange of experience in education. 

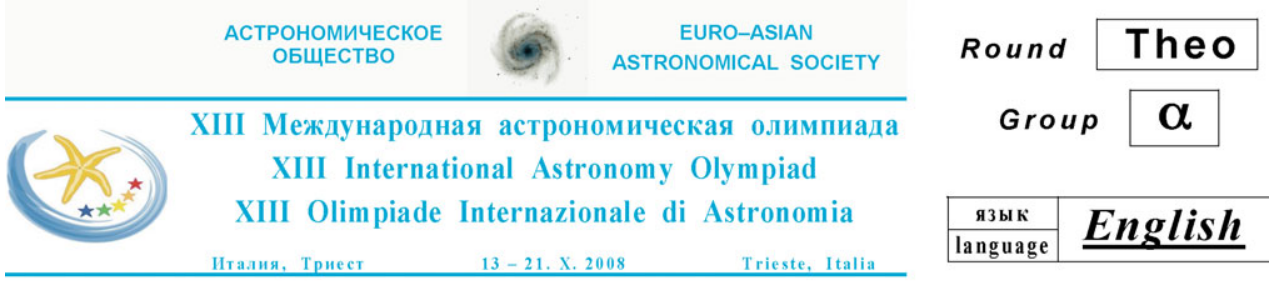

Theoretical round. Problems to solve

1. Atoll. There is an atoll on the Earth equator, from the highest point of which the Polar star appears as circumpolar. Atmospheric attenuation to be neglected. Other effects should be taken into account. Find the altitude of the atoll.

2. Absolutely black cat. Maybe you paid attention to that there are group of cats living in Grignano, to the right from the ICTP guesthouse, including four of them looking like absolutely black ones. Estimate the absolute bolometric stellar magnitude $\mathrm{M}_{\mathbf{a b e}}$ of an absolutely black cat (abc) as it is an absolutely black body.

3. Great opposition. You know that there are Great oppositions of Mars for citizens of Earth. The magnitude of Mars were even $-2^{\mathrm{m}} .9$ during some of the very famous of these oppositions (like in August 27, 2003). But there are also Great oppositions of Venus for citizens of some planet(s). At what planet(s) is this possible? Find the apparent stellar magnitude of Venus visible from

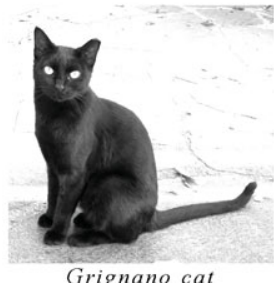

Grignano cat Photo 14.10.2008 every such planet (or this planet) at such Great oppositions.

4. Jump of bear. The beginning of XXI century. One may find in guidebooks about Spitsbergen a phrase that "a polar bear jumps 8 meters without warning". The middle of XXVI century. In order for settling remote areas of Solar system by fauna, biologists plan to deliver polar bears from Spitsbergen onto ice asteroids of the Kuiper belt. However, physicists warn, that some jumping bears can become independent objects of the Kuiper belt. Estimate, on asteroids of what sizes (diameters) it is possible to place polar bears comfortably from

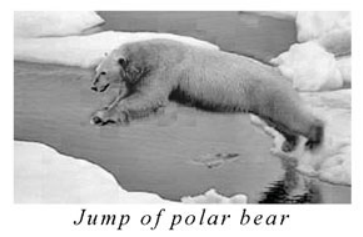
the physicists' point of view. You should give an answer in form of formula-inequality.

5. Alternative theory. The theory of an expanding Universe is the most popular and most believable modern cosmological model. They say more than $85 \%$ of astrophysicists follow this theory without any hesitance. Nevertheless some alternative theories exist as well. One of these theories proposes that the Universe is stable and the cosmological red shift appears not due to the Doppler effect, but due to "aging of photons", i.e. the energy of every photon becomes smaller according to the law $E=E_{0} \cdot 2^{-t / T o}$, where $E_{0}$ $=$ initial energy at the appearance of a photon, $\mathrm{t}=$ time of life since appearance and $\mathrm{T}_{0}=$ the so-called halfdecay period of a photon, analogous of the half-decay parameter in nuclear physics. Estimate the value of the half-decay period $\mathrm{T}_{0}$ (in years) in the model of "aging of photons" that corresponds to the value of the Hubble constant $\mathrm{H}_{\mathbf{0}}=70 \mathrm{~km} / \mathrm{s} / \mathrm{Mpc}$ in the model of expanding Universe.

Italia, Trieste

2008

Трнест, Италия

Figure 2. An example of problem sheet (theoretical round, IAO 2008).

\section{Finances}

The Olympiad needs a minimum about 1300 Euro per participant (for organising IAO in Europe). As usual $1 / 3$ of these expenses is taken from the teams as participating fees and $2 / 3$ from the institutions of the host country. Additionally teams have to pay travel expenses. Some countries cannot arrive only due to financial problems.

\section{Olympiad and Ministries of Education}

Olympiads in most of the countries exist as an activity of the Ministries of Education. The Ministries of Education consider the Olympiads as a stage of education "in subjects". 
There is no "astronomy" subject in the schools of most of the countries. So in many countries Ministries of Education "do not recognise" Astronomy Olympiads.

\section{National Astronomy Olympiads}

The IAO has stimulated founding and organizing National Astronomy Olympiads. Before 1996 the National Astronomy Olympiads took place only in 4 countries. Now more than 30 countries organize National Olympiads or Competitions in Astronomy.

\section{Rôle of Olympiads in Education}

National Astronomy Olympiads keep, support and improve the astronomy education in schools.

\section{Contacts and further information}

Website of the IAO: wwww.issp.ac.ru/iao 